\title{
EQUATIONS WITH DIRICHLET BOUNDARY NOISE
}

\author{
DALE ROBERTS
}

(Received 12 September 2012)

\begin{abstract}
2010 Mathematics subject classification: primary $60 \mathrm{H} 15$.
Keywords and phrases: stochastic partial differential equations, boundary value problems, weighted Lebesgue spaces, gamma-radonifying operators.
\end{abstract}

Stochastic partial differential equations (SPDEs) arise naturally as models for dynamical systems modelled by partial differential equations subject to random influences. As such, a large body of literature has appeared on the study of SPDEs and their more abstract formulation as stochastic evolution equations (SEEs) over the last 30 years (see, for example, $[2,5]$ ). However, sometimes these random perturbations affect a complex system not only inside the physical medium but also at its physical boundary, resulting in 'boundary noise'. This situation not only is interesting from a mathematical point of view but also occurs in a variety of applications (for example, air-sea interactions on the ocean surface, heat transfer in a solid in contact with a fluid, chemical reactor theory, and colloid and interface chemistry); see [6].

The aim of this thesis is to develop a more general theory of stochastic partial differential equations with boundary noise and, in particular, to study the question of when we can obtain function-valued solutions in the case of Dirichlet boundary conditions. Function-valued solutions are of particular importance in applications as they are the first step in designing well-posed numerical approximations of solutions.

An example of the boundary noise problem is as follows. On an open domain $O \subset \mathbb{R}^{n}$ with boundary $\partial O$, we can consider the parabolic SPDE given by

$$
\begin{cases}\frac{\partial u}{\partial t}=\mathcal{A} u+f(u)+g(u) \dot{W}_{1}(t) & \text { in }(0, T] \times O, \\ \mathcal{B} u=\sigma(x) \dot{W}_{2}(t) & \text { on }(0, T] \times O, \\ u(0, \cdot)=u_{0} & \text { in } O,\end{cases}
$$

Thesis submitted to the University of New South Wales, August 2011. Degree approved, January 2012. Supervisor: Anthony Dooley.

(C) 2012 Australian Mathematical Publishing Association Inc. 0004-9727/2012 \$16.00 
where $\mathcal{A}$ is an elliptic second-order differential operator and $\mathcal{B}$ is a differential operator given, for example, by $\mathcal{B} u:=u$ (Dirichlet) or $\mathcal{B} u:=v \cdot \nabla u$ (Neumann) where $v$ is the outward-point normal vector field on $\partial U$. Using $\dot{u}$ to denote the formal time derivative of $u$, the 'white noise' processes $\left(\dot{W}_{1}(t)\right)_{t \geq 0}$ and $\left(\dot{W}_{2}(t)\right)_{t \geq 0}$ are given in terms of the independent cylindrical Wiener processes $\left(W_{i}(t)\right)_{t \geq 0}, i=1,2$, taking values in the Hilbert spaces $L^{2}(O)$ and $L^{2}(\partial O)$, respectively. Following [2-4], the SPDE (1) can be reformulated as the SEE

$$
d U(t)=(\underline{A} U(t)+F(U(t))) d t+G(U(t)) d W_{1}(t)+\Pi_{\lambda} \Sigma d W_{2}(t),
$$

with initial condition $U(0)=u_{0}$. SEEs of the form (2) are considered in a Hilbert space framework in [3-5].

In our thesis, we propose a more general theory where $U(t)$ takes values in a Banach space $X$. As such, given Banach spaces $X$ and $\partial X$ and separable Hilbert spaces $H$ and $\partial H$, in (2) we assume that $\underline{A}: \mathcal{D}(\underline{A}) \subset X \rightarrow X, F: \mathcal{D}(F) \subset X \rightarrow X$, $G: \mathcal{D}(G) \rightarrow \mathcal{L}(H, X), \Sigma \in \mathcal{L}(\partial H, \partial X)$, and $\Pi_{\lambda}: \mathcal{D}\left(\Pi_{\lambda}\right) \rightarrow X$ for some $\lambda \geq 0$. Extending the theory to Banach spaces allows us to consider, for example, the case $X=L^{p}(O)$ with $1 \leq p \leq \infty$. Then the correspondence between (1) and (2) is given by $U(t)(x)=$ $u(t, x)$ for $(t, x) \in(0, T] \times O, \underline{A} u:=\mathcal{A} u$ for $u \in \mathcal{D}(\underline{A})=W^{2, p}(O), F(v)(x)=f(v(x))$ for $x \in O, G(v)(x)=g(v(x))$ for $x \in O,(\Sigma v)(x)=\sigma(x) v(x)$, and assuming that the elliptic boundary value problem

$$
\mathcal{A} v=\lambda v \quad \text { in } O, \quad \mathcal{B} v=w \quad \text { on } \partial U
$$

has a unique solution $v \in L^{p}(O)$, we define $\Pi_{\lambda} w:=v$. When $\mathcal{B} u:=u$, we call $\Pi$ the Dirchlet map of $w$.

To date, only one-dimensional domains had been explicitly studied. The case where $O=(0,1)$ and $X=L^{2}(O)$ was considered by Da Prato and Zabczyk [3], who showed that if one considers the heat equation on $O=(0,1)$ with white noise boundary conditions then a function-valued mild solution can be obtained in the Neumann case but not in the Dirichlet case. Alós and Bonaccorsi [1] revisited the Dirichlet problem for a parabolic SPDE (again, one-dimensional) using an analytic approach and showed that function-valued solutions can be obtained on the half-space $[0, \infty)$ if considered in the space of functions $f: \mathbb{R}_{+} \rightarrow \mathbb{R}$ such that

$$
\int_{0}^{\infty}|f(x)|^{p}\left(x^{p-1+\gamma} \wedge 1\right) d x<\infty,
$$

for some choice of $0<\gamma<1$ and $p \geq 2$. One should note that spaces of the form (4) allow solutions to blow up near the boundary $x=0$. Using our Banach space approach, we reformulate this result in our abstract Banach space framework as (2) and show that solutions in the Dirichlet case can be obtained in weighted spaces in dimensions higher than one. Unfortunately, we also show that these solutions lose their trace relationship (and thus, well-posedness) with the boundary data in dimension two and higher. This 
suggests looking at alternative approaches to (3) for mapping the stochastic boundary data into the interior of the domain.

In this direction, we explicitly study the Dirichlet map of random boundary data in dimension two and extend some classic harmonic analysis results on the unit disk $\mathbb{D}$ with boundary $\mathbb{T}$ to the stochastic setting. This leads us to work with harmonic Hardy spaces and to consider radial and non-tangential convergence towards the boundary, to obtain an interesting representation theorem, and to finally reconnect our results with weighted spaces of the form (4). We obtain the interesting characterisation that we can obtain Gaussian random variables in the harmonic Hardy space $h^{2}(\mathbb{D})$ if and only if the stochastic boundary data on $\mathbb{T}$ is not Gaussian white noise.

Returning to the problem of well-posedness for the Dirichlet map, we conclude that harmonic Hardy spaces are too small to consider the white noise boundary data. Therefore, what is the appropriate space? We consider this question from two angles. First we take a larger space, specifically the Bloch space, and show that it may be an appropriate place to consider these dynamics. We then extend Makarov's law of the iterated logarithm to our stochastic case which gives a rate of blow-up near the boundary of our harmonic random field. We conclude that although the random Bloch dynamics are very interesting, we still do not have a clear relationship with the boundary data, only an 'inside-out' viewpoint. This inspires an 'outside-in' approach whereby, due to a simple representation, we start with the white noise data on the boundary and, moving inwards, we show rates of blow-up near typical and exceptional points of the white noise process which is represented by a Brownian motion in this case. Finally, we show how these concepts may be extended to higher dimensions to provide a framework for understanding the local spatial dynamics of SPDEs.

\section{References}

[1] E. Alòs and S. Bonaccorsi, 'Stochastic partial differential equations with Dirichlet white-noise boundary conditions', Ann. Inst. H. Poincaré Probab. Statist. 38(2) (2002), 125-154.

[2] G. Da Prato and J. Zabczyk, Stochastic Equations in Infinite Dimensions, Encyclopedia of Mathematics and its Applications, 44 (Cambridge University Press, Cambridge, 1992).

[3] G. Da Prato and J. Zabczyk, 'Evolution equations with white-noise boundary conditions', Stoch. Stoch. Rep. 42(3-4) (1993), 167-182.

[4] B. Maslowski, 'Stability of semilinear equations with boundary and pointwise noise', Ann. Sc. Norm. Super. Pisa Cl. Sci. (4) 22(1) (1995), 55-93.

[5] S. Peszat and J. Zabczyk, 'An evolution equation approach', in: Stochastic Partial Differential Equations with Lévy Noise, Encyclopedia of Mathematics and its Applications, 113 (Cambridge University Press, Cambridge, 2007).

[6] D. Roberts, 'Stochastic partial differential equations with noise entering through the boundary', Preprint, 2012.

DALE ROBERTS, Centre for Mathematics and its Applications, Australian National University, Canberra, ACT 0200, Australia e-mail: dale.roberts@anu.edu.au 